\title{
Polymerase chain reaction to search for Herpes viruses in uveitic and healthy eyes: a South African perspective
}

\author{
Debbie Laaks ${ }^{1}$, Derrick P Smit ${ }^{1}$, Justin Harvey ${ }^{2}$
}

\begin{abstract}
1. Division of Ophthalmology, Department of Surgical Sciences, Faculty of Health Sciences, University of Stellenbosch, South Africa

2. Center for Statistical Consultation, University of Stellenbosch, South Africa
\end{abstract}

\begin{abstract}
:
Objective: To analyse aqueous polymerase chain reaction (PCR) results in patients diagnosed with undifferentiated uveitis and determine prevalence of herpesviridae in non-uveitic patients undergoing routine cataract extraction.

Design: Retrospective comparative case series and prospective cross-sectional study.

Subjects: 72 patients with idiopathic uveitis and 57 surgical patients.

Methods: Diagnostic aqueous paracentesis with PCR testing for 6 herpes viridae in uveitic patients. Anterior chamber paracentesis immediately pre-operative in the prospective arm, with PCR testing.

Results: In the retrospective review we had a $47.2 \%$ positive PCR yield. Data analysis revealed a statistically significant correlation between a positive yield and being HIV+ $(\mathrm{p}=0.018)$; between an EBV + yield and being HIV $+(\mathrm{p}=0.026)$ and a $\mathrm{CMV}+$ result and being HIV $+(\mathrm{p}=0.032)$. Posterior uveitis $(\mathrm{p}=0.014)$ and symptoms $<30$ days $(\mathrm{p}=0.0014)$ had a statistically significant yield. In the prospective arm of the study: all 57 patients were HIV- and all aqueous samples were negative for the 6 herpesviridae.

Conclusion: We recommend PCR testing for Herpesviridae as a safe second line test for patients with undifferentiated uveitis. We were unable to establish prevalence and suggest that the idea of a commensal herpes virus is unlikely if the blood-ocular barrier is intact.
\end{abstract}

Keywords: Polymerase chain reaction, Herpes viruses, uveitis, healthy eyes, South Africa

DOI: http://dx.doi.org/10.4314/ahs.v15i3.7

Cite as: Laaks D, Smit DP, Harvey J. Polymerase chain reaction to search for Herpes viruses in weitic and healthy eyes: a South African perspective. Afri Health Sci. 2015;15(3):748-54. doi: http:// dx.doi.org/10.4314/ahs.v15i3.7

\section{Introduction}

Uveitis is internationally classified according to the anatomical locus of infection: anterior (the anterior segment of the eye), intermediate (anterior vitreous and par plana region), posterior (retina, optic nerve, vasculature and posterior vitreous) and pan uveitis (involving all three anatomical locations). Anterior uveitis is the most common. Etiology varies widely according to the anatomical classification, age, immune status, pre-existing auto-immune diseases and exposure to toxins. Causes can be divided into immune-mediated, infectious (viral, bacterial, parasitic, mycobacteria), associated with
Corresponding author:
Debbie Laaks,
Division of Ophthalmology,
Department of Surgical Sciences,
Faculty of Health Sciences,
University of Stellenbosch, South Africa
Email: docdebbie@vodamail.co.za

systemic diseases, drug related, a white-dot syndrome or masquerade syndrome (neoplastic and non-neoplastic). Prevalence studies often underestimate the cases of uveitis as many cases are undiagnosed, misdiagnosed or may be mild and patients do not seek medical attention. Prevalence is also dependant on the patient population group under question (rural vs urban, HIV prevalence in the community etc.). Despite advances in laboratory testing, uveitis still poses many diagnostic challenges. Routine management involves a detailed history, complete ocular examination, a physical examination and targeted baseline blood investigations. Laboratory workup may include the following tests: syphilis serology, serum Angiotensin Conversion Enzyme, HLA-B27, HIV, Erythrocyte sedimentation rate and a full blood count. A chest X-ray is done and in selected cases sacro-iliac imaging. If the serum and radiological tests do not yield a definitive diagnosis, Cunningham et al suggest that these patients be classified as having idiopathic uveitis. ${ }^{1}$ More recently Jabs et al suggested the term "undifferentiated" should be used in uveitis that lacks an established non-infectious or infectious diagnosis. ${ }^{2}$ Polymerase chain reaction (PCR) testing of the aqueous 
humor has proven to be both a rapid and an accurate adjunct in the diagnosis of infectious uveitis. ${ }^{3,4}$ It can detect herpes simplex virus 1 and 2 (HSV) as well as varicella zoster virus (VZV), which accounts for up to $10 \%$ of all uveitis cases seen at tertiary level referral centers. $^{5}$ Epstein-Barr virus (EBV), cytomegalovirus (CMV) and human herpes virus 6 (HHV6) can also be detected by using PCR. 6 ,7

It is vital to remember that the presence of DNA or RNA from an infectious agent does not prove causality. ${ }^{4} \mathrm{CMV}$, for example, establishes latent infection in myeloid progenitor cells and detection of CMV DNA may therefore cause false positive results. EBV is characterized by latency in $\mathrm{B}$ and $\mathrm{T}$ lymphocytes andepithelial cells with the added ability to induce lymphoproliferation. Studies detecting EBV DNA in non-inflamed cadaveric ocular tissues and aqueous humor of immunocompetent donors indicate a broad anatomic distribution. Ongkosuwito et al documented an increased presence of Epstein-Barr virus DNA in ocular fluid samples from HIV-negative immunocompromised patients where another infectious agent had been identified as the cause of the uveitis (VZV, HSV, CMV + Toxoplasma gondii). ${ }^{8}$ A similar finding in our tertiary level unit prompted a review of the PCR yield in our uveitic patients .

Our study consisted of both a prospective and a retrospective component. In our retrospective study of a cohort of "idiopathic/undifferentiated uveitis" we aimed to: 1) analyze the PCR findings of herpesviridae, 2) determine whether a correlation exists between HIV status and PCR findings, 3) explore correlations between CD4+ count and PCR yield, 4) consider clinical features (retinitis, vasculitis and optic nerve involvement) and PCR yield and 5) probe correlations between onset of symptoms and PCR yield. This would better characterize established (HSV 1+2, VZV and CMV) and emerging (EBV and HHV6) forms of viral uveitis. The prospective arm was designed to determine the prevalence of herpesviridae in otherwise healthy eyes of patients undergoing routine cataract surgery and to review their serum HIV results. This was not an age/gender matched control group to compare to the retrospective group. We were eager to gain insight into the commensal status of these viruses in non-uveitic eyes, if any, in our local population. The results would hopefully allow us to make recommendations in the management of our challenging uveitis cases and more accurately inter- pret the PCR results in our patient population.

\section{Methods}

We conducted a retrospective evaluation of vitreous fluid samples for six herpetic viruses from a list of all aqueous samples received by the Tygerberg hospital Virology Department, from 01 January 2011 to 31 December 2012. Patient folders were then reviewed to identify patients with a diagnosis of an "undifferentiated" uveitis which was defined as active intraocular inflammation with a negative serological and radiological work-up on initial targeted testing. ${ }^{2}$ Patient characteristics and clinical features of the uveitis were also recorded. The anatomical classification adhered to the Standardization of Uveitis Nomenclature (SUN) Working Group guidelines. Aqueous samples from this group of patients were obtained in the out-patient department or in theatre after informed consent was taken. Strict aseptic techniques were employed: patients received topical anesthesia, followed by cleaning with povidone-iodine $5 \%$ solution. Thereafter, a sterile lid speculum was used and the anterior chamber paracentesis made with a 28 -gauge $12.7 \mathrm{~mm}$ needle. On average a volume of $0.1 \mathrm{ml}-0.2 \mathrm{ml}$ aqueous humor was removed. Post-procedure another drop of povidone-iodine $5 \%$ was instilled.

Patients for the prospective arm of the study were enrolled from a single health facility in Cape Town during consultations between September 2012 and January 2013. This group comprised a sample of 57 patients consenting to elective cataract surgery at Tygerberg Hospital, HIV testing and aqueous humor sampling. Inclusion criteria were: a) no active intraocular inflammation on biomicroscopic examination; b) no evidence of previous intra-ocular inflammation on history or biomicroscopic examination; c) > 18yrs old; d) normal fundus examination pre-operative or day 1 post-op if the cataract precluded a good view of the fundus pre-operative. Any evidence of previous intraocular inflammation, vitreo-retinal pathology and objection to serum HIV testing or aqueous sampling excluded patients from the study. On the day of surgery patients received a local anaesthetic block, were cleaned with a povidone-iodine $5 \%$ solution and draped as per routine strict sterile technique. Initial entry into the anterior chamber was made with a 28 -gauge needle on an insulin syringe to extract $0.1 \mathrm{ml}-0.2 \mathrm{ml}$ of aqueous fluid just anterior to limbal blood vessels. The same sample site was then extended and used as a port during the rest of the cataract surgery. After comprehensive 
pre-test counselling serum samples for HIV were obtained. Samples were processed by the National Health Laboratory Services (NHLS) Virology Department Tygerberg hospital, Cape Town, South Africa. There was strict adherence to the cold chain during transportation of the aqueous samples to the laboratory. Patients who tested positive on the confirmatory HIV test received extensive post-test counselling and a further serum sample was taken to determine their CD4+ count and viral load. They were then referred to their nearest nationally accredited Community Health Center for highly active anti-retroviral therapy (HAART) if their CD4+ count was less than 350 cells $/ \mathrm{mm}^{3}$. Patients with a positive yield on aqueous PCR were re-evaluated clinically and referred to the Medical Retina Clinic for further management.

Samples were processed using the qualitative Seeplex${ }^{\circledR}$ Meningitis-V1 ACE Detection kit (Seegene Inc., Korea) with the understanding that this method had not been validated on ocular fluids, but cerebrospinal fluid (CSF). This multiplex PCR kit employs dual priming oligonucleotide $\left(\mathrm{DPO}^{\mathrm{TM}}\right)$ technology to detect 6 viruses (HSV-1, HSV-2, VZV, EBV, CMV and HHV-6). The DPO ${ }^{\mathrm{TM}}$ primer has two different priming portions which results in very specific amplification of target DNA and consistently yields high PCR specificity even under sub-optimal PCR conditions. Limits of detection (LoD) for the Seeplex ${ }^{\circledR}$ assays are: a) HSV-1 PCR: 100 genomes/ml CSF; b) HSV-2 / VZV / EBV / CMV and HHV-6 PCR: 50 genomes/ml CSF and some studies show even lower LoD. ${ }^{9}$

Blood serum samples were screened for HIV using the third-generation immunoassays Abbot AxSym ${ }^{\circledR} \mathrm{HIV}$ $\mathrm{Ag} / \mathrm{Ab}$ Combo (Abbott Laboratories, Abbott Park, IL, USA) with a sensitivity of $100 \%$ and a specificity of $99.87 \%$. Confirmatory testing was done on all positive screening samples using the VIDAS HIV DUO Ultra (bioMérieux, Marcy l'Etoile, France) fourth-generation immunoassay. This test has a sensitivity of $100 \%$ and a specificity of at least $99.5 \%$, but has the advantage of being able to detect the p24 antigen during very early HIV infection when viral loads are still low. Where applicable a Pan-leukogate CD4+ flow-based assay test was employed to calculate absolute CD4+ counts. All serum and aqueous tests were performed at our local laboratory, using routine test assays so as not to introduce a variable between the two groups. Full panel herpesviridae screening is always done on all aqueous samples, as the cost difference between full panel versus partial panel testing is minimal.

Statistical analysis of the clinical data was completed by the Centre for Statistical Consultation of the University of Stellenbosch using MS Excel and STATISTICA version 10 [StatSoft Inc. (2011) STATISTICA (data analysis software system), www.statsoft.com]. Most variables were binary in nature and are presented using frequency distributions indicating absolute and relative counts. When two categorical variables were compared Pearson's chi-square test was used or a Fisher's exact test in the case of small expected frequencies. Continuous variables such as age, CD4 count and duration since symptom onset were found to be non-normally distributed and when comparing these to PCR yield a Mann-Whitney $U$ test was used. A p-value of $p<0.05$ was used to indicate statistical significance.

The University of Stellenbosch Health Research Ethics Committee approved both the retrospective comparative case series and prospective cross-sectional study. Internationally accepted ethical standards and guidelines were respected and patient confidentiality protected.

\section{Results}

There were 72 patients in the retrospective arm of the study. No complications during specimen collection were noted in any of the medical records. Median age at time of presentation was 30.5 years. Eighty percent of the patients were between the ages of 20 and 50 years old. Females accounted for $48(66.7 \%)$ of the cohort. Of the patients, 43 had unilateral disease (59.7\%), 24 had bilateral simultaneous uveitis $(33.3 \%)$ and 5 had bilateral asynchronous (6.9\%) involvement. Clinically there were 18 cases of anterior uveitis (25\%), 1 of intermediate uveitis $(1.4 \%), 18$ of posterior uveitis $(25 \%)$ and 35 of pan uveitis $(48.6 \%)$. Thirty three participants tested negative for HIV (45.8\%) and were deemed immune-competent. Thirty nine patients were HIV positive $(54.2 \%)$. Only 14 of the $39(35.9 \%)$ were receiving highly active antiretroviral therapy (HAART) and half of these had received treatment for 2 months or less. Of the remaining $25 \mathrm{HIV}$-positive patients, 22/25 were either recently diagnosed and not yet on HAART or had defaulted treatment and in 3/25 the HIV status was unknown. The CD4+ count of 2 of the patients in the HIV positive group was not documented. Where information allowed, we reviewed the change in CD4+ count since commencing HAART (CD4+ count at na- 
dir) and the CD4+ count on presentation to our unit. months, with apparent good compliance, had a drop One patient who had been on first line therapy for 24 in her CD4+ count from 42 to 19 cells $/ \mathrm{mm}^{3}$. Table 1

\begin{tabular}{|c|c|c|c|}
\hline \multicolumn{4}{|c|}{ Table 1. Details of the HIV positive patients $n=39 / 72$} \\
\hline & Data & Median & $\begin{array}{l}\begin{array}{l}\text { Interquartile range } \\
\quad-75^{\text {th }} \text { percentile }\end{array} \\
\quad\end{array}$ \\
\hline Age (yrs) & $39 / 39$ & 29 & $25-39$ \\
\hline CD4+ Count (absolute) & $37 / 39$ & 155 & $60-260$ \\
\hline Period on HAART (in months) & $14 / 14$ & $2 \mathrm{mnths}$ & $1 \mathrm{mnth}-15 \mathrm{mnths}$ \\
\hline $\begin{array}{l}\text { Change in CD4+ count (absolute) since } \\
\text { starting HAART and presenting }\end{array}$ & $8 / 14$ & +52 & $42.5-94.5$ \\
\hline
\end{tabular}

summarizes further patient details of the HIV positive group.

From the total of 72 patients, 34 PCR samples had a positive yield $(47.2 \%)$, detecting 36 herpesviridae: $\mathrm{HSV} 1=1 ; \mathrm{HSV} 2=4 ; \mathrm{VZV}=11 ; \mathrm{EBV}=12 ; \mathrm{CMV}$ $=5 ;$ HHV6 $=3$. Two HIV positive patients, both with a pan uveitis, tested positive for both VZV and EBV. Table 2 gives patient and clinical characteristics of the positive PCR results. Data analysis revealed a statistically significant association between a positive PCR yield and being HIV+ $(\mathrm{P}=0.0018)$; between an EBV positive yield and being HIV+ $(\mathrm{P}=0.026)$ and a CMV pos-

\begin{tabular}{|c|c|c|c|c|c|c|}
\hline \multicolumn{7}{|c|}{ Table 2. Clinical Characteristics of Positive PCR yield } \\
\hline HIV status & HSV1 (1) & HSV2 (4) & VZV (11) & EBV (12) & CMV (5) & HHV6 (3) \\
\hline HIV Negative & 1 & 2 & 3 & 2 & & 2 \\
\hline HIV Positive & & 2 & 8 & 10 & 5 & 1 \\
\hline Classification & HSV1 (1) & HSV2 (4) & VZV (11) & EBV (12) & CMV (5) & HHV6 (3) \\
\hline Anterior Uveitis & & & 3 & 1 & & 1 \\
\hline Intermediate & & & & 1 & & \\
\hline Posterior Uveitis & & 1 & 2 & 4 & 4 & 2 \\
\hline Panuveitis & 1 & 3 & 6 & 6 & 1 & 2 \\
\hline
\end{tabular}

itive result and being HIV+ $(\mathrm{P}=0.032)$. VZV indicated a trend towards association $(\mathrm{P}=0.179)$.

Patients with posterior uveitis had a significantly higher PCR yield $(\mathrm{P}=0.014)$ and CMV was found to be an important cause of posterior uveitis $(\mathrm{P}=0.0032)$.
In contrast to previous reports from elsewhere, retinitis $(\mathrm{P}=0.168)$, vasculitis $(\mathrm{P}=0.181)$ and optic nerve involvement $(\mathrm{P}=0.769)$ showed no relationship to a positive PCR yield ${ }^{3}$ in our study. A combination of all three clinical features was also not noteworthy. No as- 
sociation was found between gender and the anatomical classification of the uveitis.

A CD4+ count of less than 100 showed a trend towards association with a positive PCR yield, but this was not statistically significant $(\mathrm{P}=0.0588)$. Boxplot 1 shows the variability of $\mathrm{CD} 4+$ counts when coupled with yield. Patients who presented within 30 days since the onset of symptoms were more likely to acquire a definitive infectious diagnosis $(\mathrm{P}=0.0014)$.

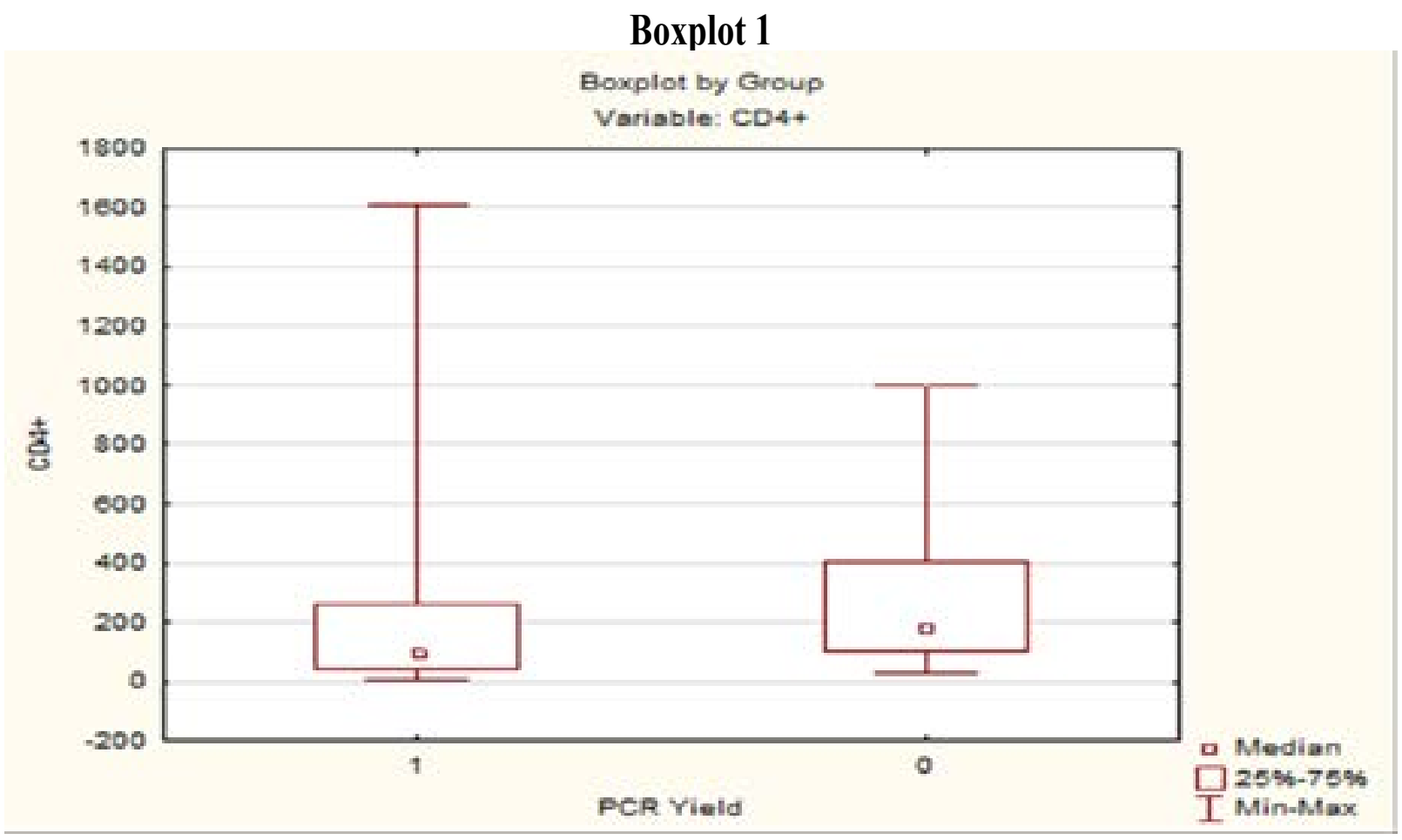

Boxplot 2 shows the distribution of duration of symptoms and the PCR results. Two of our PCR negative patients were identified as Immune recovery uveitis.

The prospective arm of the study enrolled fifty-seven patients. No intra-operative complications were record- ed and all samples were suitable for analysis. Median age was 69 years old (lower quartile $=65.8$ years; upper quartile $=71.2$ years). Fifteen males constituted $26.3 \%$ of the group. All 57 patients were HIV negative and all aqueous samples were negative for the 6 herpesviridae. We were thus not able to establish prevalence.

\section{Boxplot 2}

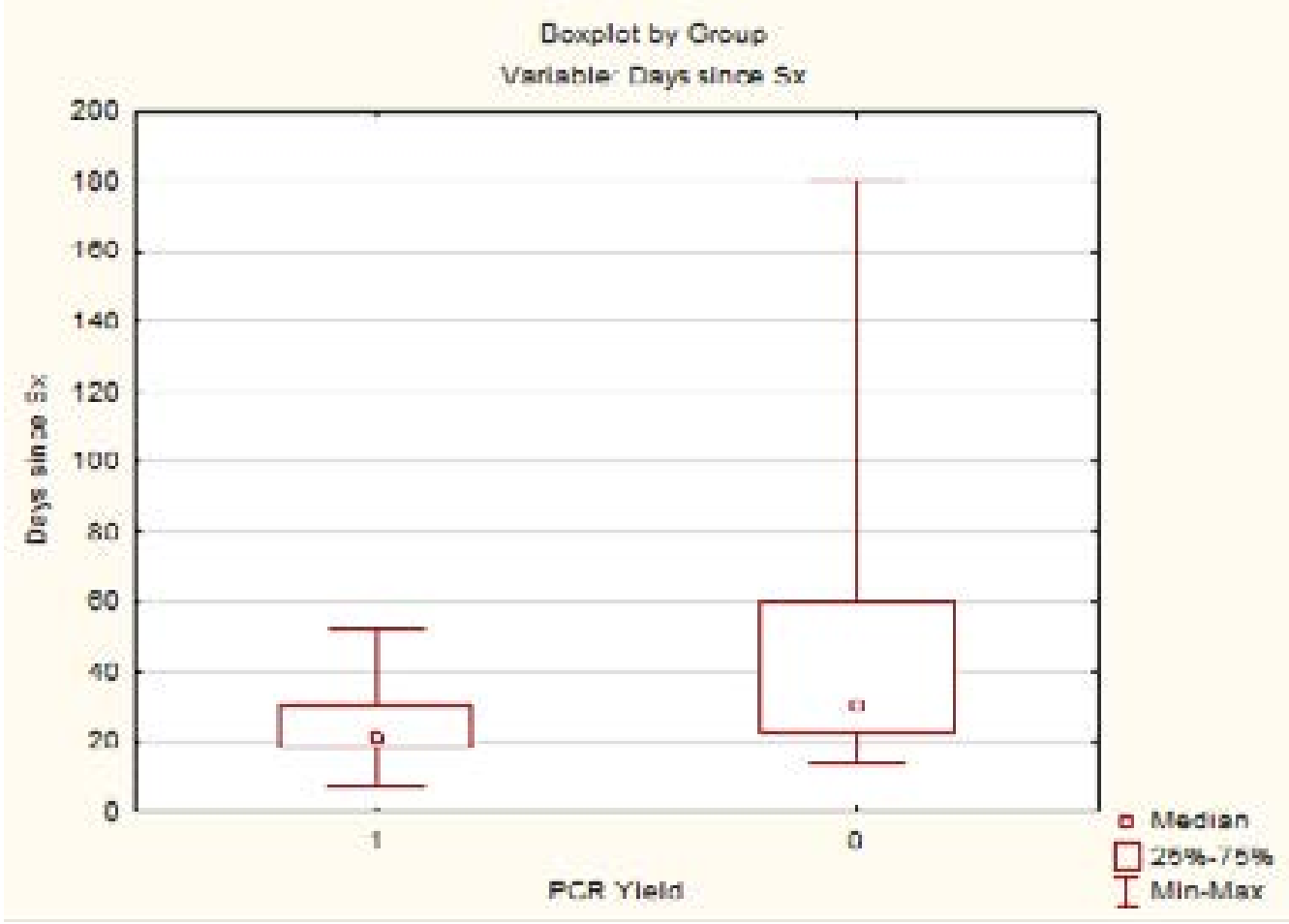




\section{Discussion}

Polymerase chain reaction analysis of viral DNA allows rapid diagnosis from very small ocular samples. In the tertiary referral center where the study was performed it has been used more frequently over the last four years to assist in the diagnosis of infectious uveitis. Analysis of aqueous humor specimens has been proven to be a safe and useful adjunct to a detailed history and thorough clinical examination. ${ }^{10}$ Collection of the sample was remarkably well tolerated and did not result in any complications in these two cohorts of patients. The median age of presentation for the uveitic group was 30.5 years and these patients are more inclined to have a non-syneritic vitreous body thereby increasing the likelihood of a 'dry tap' on needle aspiration. Anterior chamber sampling thus improves the probability of acquiring fluid for testing in a younger cohort. In cases with bilateral ocular involvement specimens were always collected from the eye with the worse visual acuity.

Until recently there was little published data on PCR analysis of ocular fluids to identify infective causes of uveitis from Sub-Saharan Africa. In a study analysing viral, parasitic and mycobacterial PCR of ocular fluids Scheepers et al noted that PCR confirmed the clinical diagnosis in 35\% and altered the initial clinical diagnosis in $23 \%$ of the patients with a posterior uveitis. Thirty-nine percent of the undifferentiated posterior uveitis cases had a definitive diagnosis after PCR testing. Their cohort also had a high percentage of HIV+ patients and they documented about 50\% qualifying for HAART, but not yet on treatment. ${ }^{11}$ In the retrospective arm of our study $35 \%$ of the patients were eligible for, yet not receiving antiretroviral therapy; despite a robust rollout of the Anti-retroviral program in South Africa.

The retrospective review allowed us to identify patients in our local population who would benefit the most from aqueous herpetic screening. Harper et al identified 6 factors that increased the chances of a positive yield on PCR testing. ${ }^{3}$ Our study correlated with 3 : immunocompromise, early presentation and posterior uveitis. We were unable to prove a statistically significant correlation between clinical presentation (retinitis, optic nerve involvement and vasculitis) and a positive PCR yield. We noted that a positive yield was independent of the patients CD4+ count, but showed a trend to be more significant in patients with lower CD4+ counts. Studies with larger cohorts may confirm this.

Early presentation was defined as: symptoms for less than 30 days. We attempted to define a more tempo- ral relationship to the disease process; this was slightly different to Harper's definition of: testing within 2 weeks of presentation. The vast geographic size of the area served by our eye unit and the poverty within our patient base pose many challenges to gaining access to tertiary level ophthalmic care. As a result there may be some delay in presentation and difficulty in reliable follow-up. In selected cases we might decide to do an aqueous tap at the time of initial targeted testing, store this sample and send for formal analysis should the screening be negative. Our definition of "early presentation" is thus independent of socio-economic circumstances and availability of sample testing and more dependent on disease course. The extended period of detecting DNA post infection has been described previously using quantitative PCR to evaluate viral loads of VZV DNA in-patients undergoing treatment for acute retinal necrosis. There was a significant decrease in viral load (>102 copies $/ \mathrm{ml}$ ) at 50 days after commencing treatment with antiviral drugs. ${ }^{12}$ Confirming that PCR is still of value in patients previously thought to be 'presenting late'.

The role of EBV as a cause for uveitis is unclear. Twelve of our samples tested positive for EBV and there was a statistically significant association between the detection of EBV in aqueous humor and HIV infection. Various studies, utilising serum IgG ELISA assays, have shown that EBV is hyperendemic in both HIV-seronegative and HIV-seropositive patients. A study in Ghana noted that the seroprevalence of EBV was statistically significantly higher $(p<0.001)$ in HIVAIDS patients compared to HIV-seronegative healthy blood donors. ${ }^{13}$ This poses the question whether we are merely detecting leakage from the systemic circulation through an abnormally permeable blood-ocular barrier or detecting active replication within the eye ? EBV was first described in 1990 as a cause of uveitis in 3 patients, validated by EBV $\operatorname{IgG}$ antibodies in the aqueous ${ }^{14}$ and was once demonstrated histologically in an enucleated uveitic eye. ${ }^{15}$ In 2009 Kim et al did a review of cases in the literature and highlighted EBV's propensity to being a co-pathogen ${ }^{16,17,18}$ most commonly with VZV. This finding is supported by two cases in this study that tested positive for both EBV and VZV. Our study made use of a qualitative PCR technique and therefore cannot comment on the quantum of EBV present inside the eye. Future studies measuring the EBV viral load by quantitative PCR as well as possibly calculating an EBV Goldmann-Witmer Coefficient to look for antibody production against the virus may help to implicate or exonerate EBV as a pathogen. 
To our knowledge the prospective arm of the study is the largest cohort of healthy eyes where only aqueous samples were used and full panel Herpes viridae testing was done on all specimens. We were unable to establish prevalence and thus propose that the idea of a commensal herpes virus in the aqueous is unlikely if the blood-ocular barrier is intact and the individual is immune-competent. Larger studies are needed to support this theory. For example, a prospective study evaluating a cohort of HIV positive patients with normal eyes, undergoing routine surgery may reveal different results and address some of the short comings of our group.

Clinical acumen and a thorough history should never be replaced by broad-based testing. We recommend PCR testing for herpes viridae in patients as a second line test as up to $50 \%$ of these investigations may yield a causative infectious agent. HIV+ patients, patients presenting with symptoms early and patients with posterior uveitis appear to have the most to gain in our setting. Larger studies need to be conducted, but thus far no commensal herpes viruses have been identified in our non-uveitic population. The results of these two studies allow us to test more appropriately and thus institute anti-viral treatment promptly in challenging uveitis cases in our local patient population.

\section{Financial support:}

University of Stellenbosch Ophthalmology Department Funding

\section{Conflict of interest:}

No conflicting relationship exists for any author.

\section{References}

1. Cunningham ET Jr, Baglivo E. Fuchs heterochromic iridocyclitis-syndrome, disease, or both? Am J Ophthalmol 2009;148:479-81 PubMed .

2. Jabs DA, Busingye J. Approach to the Diagnosis of the Uveitides. Am J Ophthalmol 2013;156:228-36 PubMed .

3. Harper TW, Miller D, Schiffman JC, Davis JL. Polymerase Chain Reaction Analysis of Aqueous and Vitreous Specimens in the Diagnosis of Posterior Segment Infectious Uveitis. Am J of Ophthalmol 2009;147:140-7 PubMed .

4. de Groot-Mijnes JD, de Visser L, Zuurveen S, et al. Identification of new pathogens in the intraocular fluid of patients with uveitis. Am J Ophthalmol 2010;150:62836 PubMed .
5. Siverio CD Jr, Imai Y, Cunningham ET Jr. Diagnosis and management of herpetic anterior uveitis. Int $O p h$ thalmol Clin 2002;42:43-8 PubMed .

6. Van Gelder RN. Ocular pathogens for the twenty-first century. Am J Ophthalmol 2010;150:595-7 PubMed .

7. Drancourt M, Berger P, Terrada C, et al. High prevalence of fastidious bacteria in 1520 cases of uveitis of unknown etiology. Medicine 2008;87:167-76 PubMed .

8. Chodosh J, Gan YJ, Sixbey JW. Detection of Ebstein-Barr virus genome in ocular tissue. Ophthalmology 1996;103:687-90 PubMed .

9. Ongkosuwito JV, Van der Lelij A, Bruinenberg M, et al. Increased presence of Epstein-Barr virus DNA in ocular fluid samples from HIV negative immunocompromised patients with uveitis. $\mathrm{Br} J$ Ophthalmol 1998;82:245-51 PubMed .

10.Shin SY, Kwon KC, Park JW, et al. Evaluation of the Seeplex ${ }^{\circledR}$ Meningitis ACE Detection kit for the detection of 12 common bacterial and viral pathogens. Ann Lab Med 2012;32:44-9 PubMed.

11.Cheung CMG, Durrani OM, Murray PI. The safety of anterior chamber paracentesis in patients with uveitis. Br J Ophthalmol 2004; 88:582-3.

12.Scheepers MA, Lecuona KA, Rogers G, et al. The Value of Routine Polymerase Chain Reaction Analysis of Intraocular Fluid Specimens in the Diagnosis of Infectious Posterior Uveitis. The Scientific World Journal. 2013/545149:8 pages

13. Bernheim D, Germi R, Labetoulle M, et al. Time Profile of Viral DNA in Aqueous Humor Samples of Patients Treated for Varicella-Zoster Virus Acute Retinal Necrosis by Use of Quantitative Real-Time PCR. J Clin Microbiol 2013;51:2160-6 PubMed

14. Adjei1 AA, Armah HB, Gbagbo F et al. Seroprevalence of HHV-8, CMV, and EBV among the general population in Ghana, West Africa. BMC Infectious Diseases 2008, 8:111

15. Usui M, Sakai J. Three cases of EBV-associated uveitis. Int Opbthalmol 1990;14:371-6 PubMed .

16. Freigassner, Ardjomand N, Radner H, El-Shabrawi Y. Coinfection of the retina by Epstein-Barr virus and cytomegalovirus in an AIDS patient. Am J Ophthalmol 2002;134:275-7 PubMed .

17. Kim SJ, Baranano DE, Grossniklaus HE, Martin DF. Epstein-Barr infection of the Retina: case report and review of the literature. Retinal Cases \& Brief Reports 2009;1-1

18. Lau CH, Missotten T, Salzmann J, Lightman SL. Acute Retinal Necrosis: Features, Management, and Outcomes. Ophthalmology 2007;114:756-762 PubMed 